\title{
Semiquantal analysis of hydrogen bond
}

$\operatorname{AUTHOR}(\mathrm{S})$ :

Ando, $\mathrm{K}$

\section{CITATION:}

Ando, K. Semiquantal analysis of hydrogen bond. JOURNAL OF CHEMICAL PHYSICS 2006, 125(1): 014104.

\section{ISSUE DATE:}

2006-07-01

URL:

http://hdl.handle.net/2433/49821

\section{RIGHT:}

Copyright 2006 American Institute of Physics. This article may be downloaded for personal use only. Any other use requires prior permission of the author and the American Institute of Physics. 


\title{
Semiquantal analysis of hydrogen bond
}

\author{
Koji Ando ${ }^{\text {a) }}$ \\ Department of Chemistry, Graduate School of Science, Kyoto University, Sakyo-ku, \\ Kyoto 606-8501, Japan
}

(Received 24 March 2006; accepted 11 May 2006; published online 5 July 2006)

\begin{abstract}
The semiquantal time-dependent Hartree (SQTDH) theory is applied to the coupled Morse and modified Lippincott-Schroeder (LS) model potentials of hydrogen bond. The structural correlation between the heavy atoms distance and the proton position, the geometric isotope effect, the energy of hydrogen bond formation, and the proton vibrational frequency shift are examined in a broad range of structural parameters. In particular, the geometric isotope effect is found to depend notably on the choice of the potential model, for which the LS potential gives the isotope shift of the heavy atoms distance in the range of $0.02-0.04 \AA$, in quantitative agreement with the experimental findings from assortment of hydrogen bonding crystals. The fourth-order expansion approximation to the semiquantal extended potential was confirmed to be highly accurate in reproducing the full SQTDH results. The approximation is computationally efficient and flexible enough to be applied to general models of hydrogen bond. (C) 2006 American Institute of Physics.
\end{abstract}

[DOI: $10.1063 / 1.2210477]$

\section{INTRODUCTION}

One interesting nature of hydrogen bond is the range of structural and energetic properties exhibited under different chemical conditions and in various substances. ${ }^{1-4}$ For example, the $A \cdots B$ distance in $A-\mathrm{H} \cdots B$ hydrogen bond is typically observed in the range of $2.4-3.0 \AA$, the hydrogen bond energy $2-12 \mathrm{kcal} / \mathrm{mol}$, the $A-\mathrm{H}$ bond length 0.95-1.2 $\AA$, and the $A-\mathrm{H}$ vibrational frequency around $1700-3600 \mathrm{~cm}^{-1}$. Associated with the decrease of the $A \cdots B$ distance, the hydrogen bond energy becomes larger, the $A-\mathrm{H}$ length longer, and the $A-\mathrm{H}$ vibrational frequency smaller. These correlated changes appear to bear significant clue for understanding chemical properties and functions of hydrogen bonding materials.

The $a b$ initio quantum chemical methods have been successful in reproducing many of these static properties, ${ }^{5-7}$ although after elaborate computations of the electron correlation effects with sufficiently large basis set. These calculations are still expensive enough to hinder their implementations into the condensed phase molecular simulations when sufficient statistical samplings are essential. In this sense, the empirical or semiempirical models are of practical value for widening our approaches to variety of problems. One of the most studied empirical potentials is the one proposed by Lippincott and Schroeder. ${ }^{8-12}$ The empirical valence bond model ${ }^{13}$ is also actively used in condensed phase simulation studies.

Compared to the electronic structural aspects under the adiabatic approximation, the quantum mechanical nature of the proton has been studied rather scarcely to date. The $A-\mathrm{H}$ vibrational frequency of $3000 \mathrm{~cm}^{-1}$ corresponds to the zeropoint energy of $4 \mathrm{kcal} / \mathrm{mol}$, which implies that the quantum nature is far from negligible. In particular, the zero-point

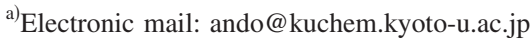

energy level in strong hydrogen bonds may be located above the potential barrier (or the convex region) such that the proton wave function is delocalized over the complex. ${ }^{14}$ This will affect the structure and dynamics in ways beyond the classical mechanical description. The quantum nature of the proton also correlates with the heavy atoms distance $A \cdots B$, as is observed in the geometric isotope effect, for which consistent treatment of the light and heavy degrees of freedom would be essential. Recent advances of the combined nuclear and electronic orbitals methods ${ }^{15-17}$ appear to be directing toward these aspects.

The primary motivation of this work is to devise a practical and efficient method to take account of the nuclear quantum aspects in hydrogen bonds. To this end, we apply the semiquantal time-dependent Hartree (SQTDH) theory developed recently. ${ }^{18,19}$ The empirical potential models are usefully employed to examine the nature of hydrogen bond in a broad range of the structural parameters. Extensive works to combine with the electronic structure calculations are planned, but are beyond the scope of this article.

The next section summarizes the SQTDH theory. The empirical potential models and their semiquantal treatments are described in Sec. III. The calculations of the structural correlations, the geometric isotope effect, the hydrogen bond energy, and the vibrational frequency shift are discussed in Sec. IV. The paper concludes in Sec. V.

A preliminary report of this work has been published. ${ }^{20}$ The novel aspects in the present article include an assessment of the fourth-order expansion approximation, comparison between the coupled Morse and modified LippincottSchroeder potentials, analysis of the wave packet widths, refinement of the parameters to better reproduce the hydrogen bond formation energy, and the examination of the vibrational frequency shift. 


\section{SQTDH THEORY}

The SQTDH theory has been outlined previously. ${ }^{19}$ It describes the wave function as a Hartree product of the squeezed coherent state Gaussian wave packets characterized by the center and width parameters. The equations of motion for the parameters are determined from the time-dependent variational principle. ${ }^{21-23}$ One of the marked features is that the equations of motion for both the center and width parameters have the form of classical Hamiltonian equations of motion. ${ }^{24-26}$ This suggests us to extend the phase space such as to include the width coordinates and their conjugate momenta in addition to the center coordinates. The wave packet dynamics is thus viewed as classical motion on an effective potential in the extended phase space. In particular, the effect of dissipative bath can be taken into account straightforwardly and in a pictorial manner. ${ }^{19}$ Another computational advantage is that the optimal stationary state wave function is directly obtained from the minimum point on the extended potential.

For simplicity of notation, we set $\hbar=1$ and assume that the coordinates $q_{1}, \ldots, q_{f}$ have been mass scaled. The trial wave function is defined with the time-dependent Hartree ansatz

$$
\begin{aligned}
\Psi\left(q_{1}, \ldots, q_{f}, t\right)= & \prod_{\alpha=1}^{f} N_{\alpha} \exp \left\{A_{\alpha}(t)\left(q_{\alpha}-x_{\alpha}(t)\right)^{2}+i p_{\alpha}(t)\right. \\
& \left.\times\left(q_{\alpha}-x_{\alpha}(t)\right)\right\}
\end{aligned}
$$

in which

$$
A_{\alpha}(t)=\frac{-1+2 i \rho_{\alpha}(t) \pi_{\alpha}(t)}{4 \rho_{\alpha}(t)^{2}} .
$$

$N_{\alpha}=\left(2 \pi \rho_{\alpha}(t)^{2}\right)^{-1 / 4}$ is the normalization factor. The timedependent parameters $x_{\alpha}(t)$ and $\rho_{\alpha}(t)$ describe the centers and widths of the wave packet. The parameters $p_{\alpha}(t)$ and $\pi_{\alpha}(t)$ are seen later to describe the conjugate momenta of $x_{\alpha}(t)$ and $\rho_{\alpha}(t)$, respectively.

The Lagrangian for the time-dependent variational theory is given by

$$
L=\left\langle\Psi, t\left|i \frac{\partial}{\partial t}+\sum_{\alpha} \frac{\partial^{2}}{2 \partial q_{\alpha}^{2}}-V\left(q_{1}, \ldots, q_{f}\right)\right| \Psi, t\right\rangle,
$$

in which $V$ is the potential. The variational condition $\delta\left(\int L d t\right) / \delta X=0$, where $X$ represents all the parameters, yields the equations of motion,

$$
\begin{aligned}
& \dot{x}_{\alpha}=\partial H_{\mathrm{ext}} / \partial p_{\alpha}, \quad \dot{p}_{\alpha}=-\partial H_{\mathrm{ext}} / \partial x_{\alpha}, \\
& \dot{\rho}_{\alpha}=\partial H_{\mathrm{ext}} / \partial \pi_{\alpha}, \quad \dot{\pi}_{\alpha}=-\partial H_{\mathrm{ext}} / \partial \rho_{\alpha},
\end{aligned}
$$

in which the extended Hamiltonian is defined by

$$
H_{\mathrm{ext}}=\sum_{\alpha=1}^{f}\left[\frac{p_{\alpha}^{2}}{2}+\frac{\pi_{\alpha}^{2}}{2}+\frac{1}{8 \rho_{\alpha}^{2}}\right]+\langle V\rangle .
$$

These direct us to investigate the quantum dynamics in an extended phase space formed by $\left(x_{\alpha}, \rho_{\alpha}, p_{\alpha}, \pi_{\alpha}\right)$. The key quantity is the extended potential

$$
V_{\mathrm{ext}}=\sum_{\alpha=1}^{f} \frac{\hbar^{2}}{8 m_{\alpha} \rho_{\alpha}^{2}}+\langle V\rangle
$$

defined in the configuration space $\left(x_{\alpha}, \rho_{\alpha}\right)$. Note that $p_{\alpha}$ and $\pi_{\alpha}$ vanish in $\langle V\rangle$ as they appear only in the phase factor of $\Psi$. Note also that $\hbar$ and the atomic masses $m_{\alpha}$ have been retrieved in the first term of Eq. (6). This term serves to broaden the wave packet by forming a potential wall growing along $\rho \rightarrow+0$, with the stronger tendency when the mass is the lighter, and vanishes in the classical limit $\hbar \rightarrow 0$.

The calculation of $\langle V\rangle$ is straightforward only in limited cases. The coupled Morse potential described in the next section is an example where a compact analytical form is available. For general potentials, the Taylor expansion of $V$ around $\Delta q_{\alpha} \equiv q_{\alpha}-\left\langle q_{\alpha}\right\rangle$ may be useful. Since $\left\langle q_{\alpha}\right\rangle=x_{\alpha}$, $\left\langle\Delta q_{\alpha}^{2}\right\rangle=\rho_{\alpha}^{2},\left\langle\Delta q_{\alpha}^{4}\right\rangle=3 \rho_{\alpha}^{4}$, and so on, we find ${ }^{19}$

$$
\begin{aligned}
\langle V\rangle= & V\left(x_{1}, \ldots, x_{f}\right)+\frac{1}{2} \sum_{\alpha=1}^{f} \frac{\partial^{2} V}{\partial q_{\alpha}^{2}} \rho_{\alpha}^{2}+\frac{1}{4} \sum_{\alpha<\beta}^{f} \frac{\partial^{4} V}{\partial q_{\alpha}^{2} \partial q_{\beta}^{2}} \rho_{\alpha}^{2} \rho_{\beta}^{2} \\
& +\frac{1}{8} \sum_{\alpha=1}^{f} \frac{\partial^{4} V}{\partial q_{\alpha}^{4}} \rho_{\alpha}^{4}+\ldots .
\end{aligned}
$$

We will assess the accuracy of the fourth-order expansion for the hydrogen bond model potentials. As the second derivative of the potential will be negative around the potential barrier region, divergence along $\rho$ may be caused if the expansion was truncated at the second order. Therefore, the fourth-order expansion would be the minimum requirement for double-well potentials. It is interesting to note that the fourth-order expansion is essentially equivalent to the QHD-2 theory ${ }^{27}$ derived via an apparently different route from mixed quantum-classical Heisenberg equations of motion.

\section{MODEL POTENTIALS AND PARAMETERS}

The Lippincott-Schroeder model describes the $A-\mathrm{H} \cdots B$ hydrogen bond complex by a sum of $A-\mathrm{H}$ and $B-\mathrm{H}$ bond potentials and nonbonded $A \cdots B$ potential. Following this idea, we have constructed an alternative form of model potential employing the Morse potential for all the three components. Although the Morse potential has been examined to be no better than the Lippincott potential for modeling covalent bonds, ${ }^{28}$ it still has an advantage in the SQTDH theory that an exact analytical form of the extended potential is available. We thereby employ the coupled Morse (CM) potential as a cornerstone for developing and testing the theory, and then proceed to the study of the Lippincott-Schroeder (LS) potential. In particular, it plays a critical role in the assessment of the fourth-order expansion approximation.

\section{A. Coupled Morse potential}

We define the coupled Morse potential by

$$
V^{\mathrm{CM}}=V_{A \mathrm{H}}^{M}+V_{B \mathrm{H}}^{M}+V_{A B}^{M}-D_{B \mathrm{H}}-D_{A B},
$$

where $V_{\mathrm{XY}}^{M}$ is the Morse function for the $\mathrm{XY}$ bond, 


$$
V_{\mathrm{XY}}^{M}=D_{\mathrm{XY}}\left[1-\exp \left(-a_{\mathrm{XY}}\left(r_{\mathrm{XY}}-r_{\mathrm{XY}}^{0}\right)\right)\right]^{2} .
$$

The parameters $D_{\mathrm{XY}}$ and $r_{\mathrm{XY}}^{0}$ are the bond dissociation energy and the equilibrium bond length, and $a_{\mathrm{XY}}$ is related to the potential well curvature. The last two terms in Eq. (8) are subtracted such that the completely dissociated complex has $V^{\mathrm{CM}}=0$ at the classical minimum.

Among the bare internuclear distances $r_{A \mathrm{H}}, r_{B \mathrm{H}}$, and $r_{A B}$, two are independent in the collinear complex. They are converted to the Jacobi coordinates ${ }^{29} r$ and $R$ that diagonalize the kinetic operators, as defined by $r=r_{A \mathrm{H}}$ and $R=r_{B \mathrm{H}}$ $+\alpha_{A} r_{A \mathrm{H}}$ where $\alpha_{A}=m_{A} /\left(m_{A}+m_{\mathrm{H}}\right)$ and $m_{X}$ denotes the mass of atom $X$. For ordinary hydrogen bonds with $m_{A} \gg m_{\mathrm{H}}$, we find $R \simeq r_{A B}$. The mass for the Jacobi coordinates are given by $m_{r}=m_{A} m_{\mathrm{H}} /\left(m_{A}+m_{\mathrm{H}}\right)$ and $m_{R}=\left(m_{A}+m_{\mathrm{H}}\right) m_{B} /\left(m_{A}+m_{\mathrm{H}}\right.$ $\left.+m_{B}\right)$. Conversely, the bare internuclear distances are retrieved as $r_{A \mathrm{H}}=r, r_{B \mathrm{H}}=R-\alpha_{A} r$, and $r_{A B}=R+\alpha_{\mathrm{H}} r$ where $\alpha_{\mathrm{H}}$ $=m_{\mathrm{H}} /\left(m_{A}+m_{\mathrm{H}}\right)$.

We will now denote the centers of the SQTDH wave packets by the same symbols $r$ and $R$, assuming that no confusion will arise between the coordinates and the parameters. The corresponding wave packet widths are denoted by $w$ and $W$. The semiquantal extended potential for $V^{\mathrm{CM}}$ is straightforwardly derived as

$$
\begin{aligned}
V_{\mathrm{ext}}^{\mathrm{CM}}(r, R, w, W) & \\
= & \frac{\hbar^{2}}{8 m_{r} w^{2}}+\frac{\hbar^{2}}{8 m_{R} W^{2}}+V_{\mathrm{ext}}^{M}\left(r, r_{A \mathrm{H}}^{0}, w^{2}, a_{A \mathrm{H}}, D_{A \mathrm{H}}\right) \\
& +V_{\mathrm{ext}}^{M}\left(R-\alpha_{A} r, r_{B \mathrm{H}}^{0}, W^{2}+\alpha_{A}^{2} w^{2}, a_{B \mathrm{H}}, D_{B \mathrm{H}}\right)-D_{B \mathrm{H}} \\
& +V_{\mathrm{ext}}^{M}\left(R+\alpha_{\mathrm{H}} r, r_{A B}^{0}, W^{2}+\alpha_{\mathrm{H}}^{2} w^{2}, a_{A B}, D_{A B}\right)-D_{A B},
\end{aligned}
$$

where

$$
\begin{aligned}
& V_{\mathrm{ext}}^{M}\left(r, r^{0}, w^{2}, a, D\right) \\
& \quad=D\left[1+e^{-2 a\left(r-r^{0}\right)+2 a^{2} w^{2}}-2 e^{-a\left(r-r^{0}\right)+a^{2} w^{2} / 2}\right] .
\end{aligned}
$$

\section{B. Lippincott-Schroeder potential}

The Lippincott-Schroeder potential for hydrogen bond is given by ${ }^{8}$

$$
V^{\mathrm{LS}}=V_{A \mathrm{H}}^{L}+V_{B \mathrm{H}}^{L}+V_{A B}-D_{B \mathrm{H}},
$$

where $V_{\mathrm{XY}}^{L}$ is the Lippincott potential ${ }^{30,31}$ for the $\mathrm{XY}$ bond

$$
V_{\mathrm{XY}}^{L}=D_{\mathrm{XY}}\left[1-\exp \left(-n_{\mathrm{XY}}\left(r_{\mathrm{XY}}-r_{\mathrm{XY}}^{0}\right)^{2} / 2 r_{\mathrm{XY}}\right)\right] \text {. }
$$

In the original paper, ${ }^{8}$ the nonbonded term was given the form $V_{A B}=A \exp \left(-b r_{A B}\right)-B / r_{A B}^{m}$, and the results of the calculations were found to be insensitive to the choice of $m$ $=1$ or 6 . Consequently, some later works omitted the term $-B / r_{A B}^{m}$ and others used the Morse function for the $V_{A B}$ part. ${ }^{10,12}$ We will follow the latter option considering the merit to have the correspondence with the CM potential. Therefore, our choice is

$$
V^{\mathrm{LS}}=V_{A \mathrm{H}}^{L}+V_{B \mathrm{H}}^{L}+V_{A B}^{M}-D_{B \mathrm{H}}-D_{A B} .
$$

We will call it a modified LS potential (or simply the LS potential hereafter).
Due to lack of a compact analytical form of $\left\langle V^{L}\right\rangle$, we employ the fourth-order expansion approximation [cf. Eq. (7)] and find

$$
\begin{aligned}
V_{\mathrm{ext}(4)}^{\mathrm{LS}}(r, R, w, W) & \\
= & \frac{\hbar^{2}}{8 m_{r} w^{2}}+\frac{\hbar^{2}}{8 m_{R} W^{2}}+V^{\mathrm{LS}}(r, R)+\frac{1}{2}\left(V_{A \mathrm{H}}^{L(2)}+\alpha_{A}^{2} V_{B \mathrm{H}}^{L(2)}\right. \\
& \left.+\alpha_{\mathrm{H}}^{2} V_{A B}^{M(2)}\right) w^{2}+\frac{1}{2}\left(V_{B \mathrm{H}}^{L(2)}+V_{A B}^{M(2)}\right) W^{2}+\frac{1}{4}\left(\alpha_{A}^{2} V_{B \mathrm{H}}^{L(4)}\right. \\
& \left.+\alpha_{\mathrm{H}}^{2} V_{A B}^{M(4)}\right) w^{2} W^{2}+\frac{1}{8}\left(V_{A \mathrm{H}}^{L(4)}+\alpha_{A}^{4} V_{B \mathrm{H}}^{L(4)}\right. \\
& \left.+\alpha_{\mathrm{H}}^{4} V_{A B}^{M(4)}\right) w^{4}+\frac{1}{8}\left(V_{B \mathrm{H}}^{L(4)}+V_{A B}^{M(4)}\right) W^{4},
\end{aligned}
$$

in which $V_{\mathrm{XY}}^{L / M(2)}$ and $V_{\mathrm{XY}}^{L / M(4)}$ are the second and fourth derivatives of $V_{X Y}^{L / M}$ with respect to $r_{X Y}$. The corresponding expansion for the CM potential $V_{\mathrm{ext}(4)}^{\mathrm{CM}}$ is obtained similarly.

\section{Parameters and computationals}

The parameters for the potentials are mostly taken from Refs. 8 and 12, and hence correspond to the $\mathrm{O}-\mathrm{H} \cdots \mathrm{O}$ hydrogen bonds. For the bonding part, we take $D_{A \mathrm{H}}$ $=110.6 \mathrm{kcal} / \mathrm{mol}, D_{B \mathrm{H}}=D_{A \mathrm{H}} / g, n_{A \mathrm{H}}=9.8 \AA^{-1}, n_{B \mathrm{H}}=n_{A \mathrm{H}} \cdot g$, and $r_{A \mathrm{H}}^{0}=r_{B \mathrm{H}}^{0}=0.97 \AA$. The parameter $g$ controls the asymmetry of the potential, and its numerical value will be speci-

(a)

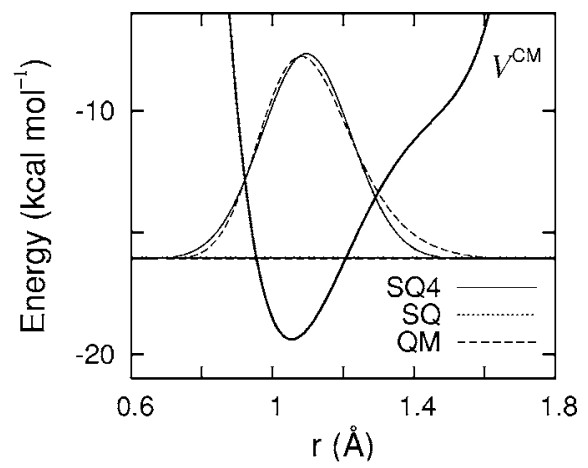

(b)

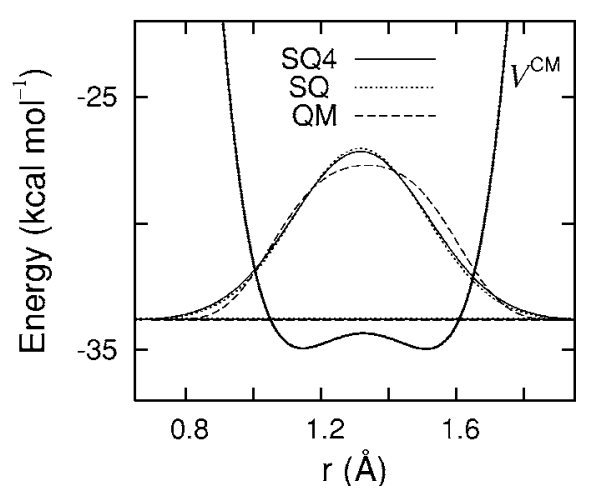

FIG. 1. The coupled Morse potential along the Jacobi coordinate $r$, and the ground state wave functions from the SQTDH calculation with the exact extended potential (dotted, labeled SQ) and the fourth-order approximation (solid, SQ4). The asymmetry parameter $g$ is 1.45 (a) and 1.0 (b), respectively. The numerical quantum solution is also included (dashed, QM). The horizontal lines are the corresponding energy levels. The amplitude of the wave functions is in arbitrary unit. 
(a)

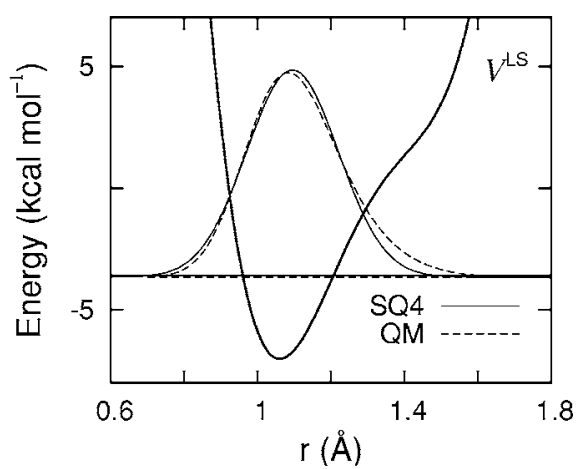

(b)

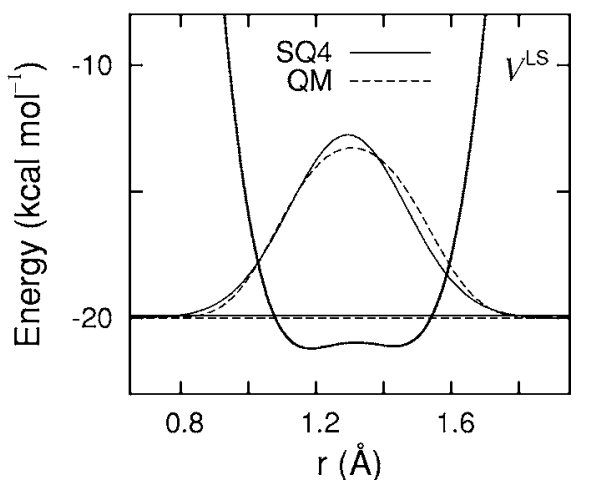

FIG. 2. Same as Fig. 1 but for the modified Lippincott-Schroeder potential. Note that the exact semiquantal solution (SQ) is unavailable for this potential, so that only the QM and SQ4 results are compared.

fied in the results. The parameters $a_{\mathrm{XY}}$ are derived via $a$ $=\sqrt{n / 2 r^{0}}$ to give the same potential curvature between $V_{\mathrm{XY}}^{M}$ and $V_{\mathrm{XY}}^{L}$ at the well bottom. For the nonbonded part, we take $a_{A B}=2.9 \AA^{-1}$ from Ref. 12. In the previous publication, ${ }^{20}$ we also used $D_{A B}=2000 \mathrm{~cm}^{-1}=5.7 \mathrm{kcal} / \mathrm{mol}$ from the same reference. However, in the course of the present work we find it overestimates the hydrogen bond formation energy by around $5 \mathrm{kcal} / \mathrm{mol}$. We have thus reoptimized it and obtained $D_{A B}=1.6 \mathrm{kcal} / \mathrm{mol}$, which gives reasonably good results as will be demonstrated later. $r_{A B}^{0}$ is taken as a variable parameter that controls the shape of the potential and hence the results in Figs. 3-8. $R_{A B}$ is thus not an external parameter but is an outcome of the calculation. (Hereafter, the $A B$ distance will be denoted by the capital $R_{A B}$.) We found monotonic but nonlinear correlation between $r_{A B}^{0}$ and $R_{A B}$.

The optimal wave packet is obtained by minimizing $V_{\text {ext }}$ in the extended coordinate space $(r, R, w, W)$. We employ the downhill simplex and the direction set methods ${ }^{32}$ for the minimization. Both methods were used to check the convergence of the results.

\section{RESULTS AND DISCUSSION}

\section{A. Accuracy of the fourth-order approximation}

Figure 1 compares the wave functions from the exact $V_{\mathrm{ext}}^{\mathrm{CM}}$ and the fourth-order approximation $V_{\mathrm{ext}}^{\mathrm{CM}}$ (SQ and SQ4 in the figure) for the CM potential. They look almost indistinguishable in both the asymmetric and symmetric cases. The quantum mechanical (QM) solutions by the renormalized Numerov method ${ }^{33,34}$ are also displayed. It is seen that the agreement of the semiquantal wave packets to the quan-
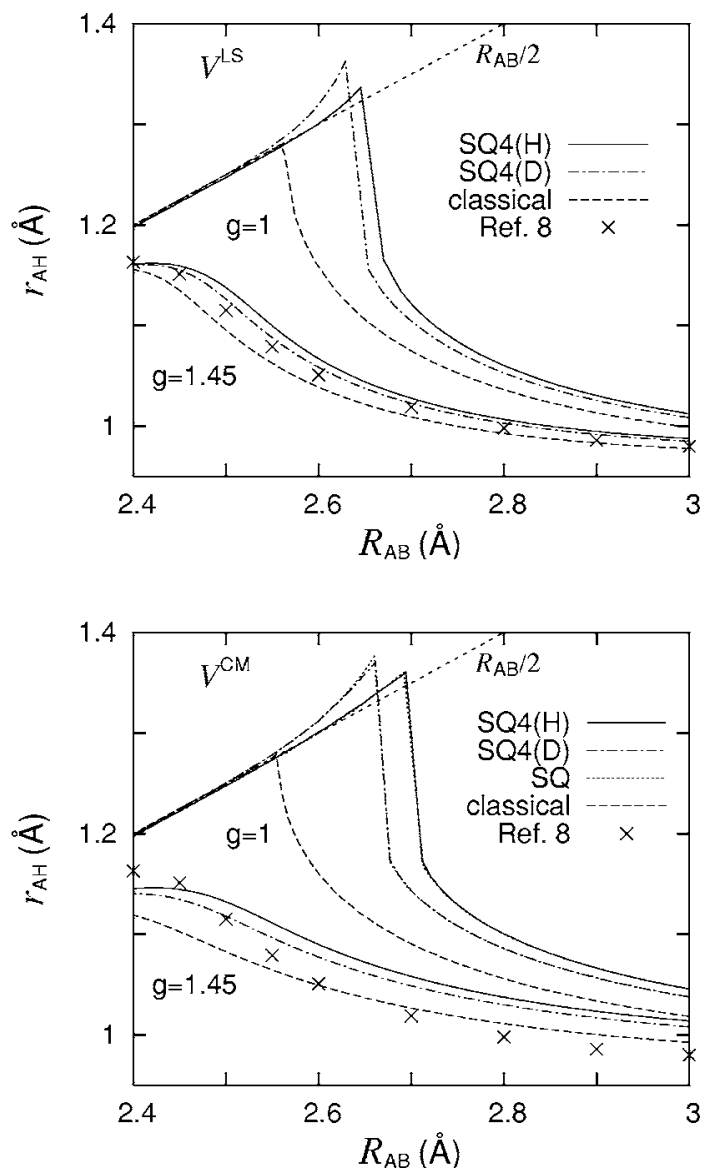

FIG. 3. The correlation between the internuclear distances $R_{A B}$ and $r_{A \mathrm{H}}$ for the modified Lippincott-Schroeder (a) and the coupled Morse (b) potentials. Solid and dash-dotted lines are from the fourth-order SQTDH (SQ4) calculations for hydrogen and deuterium, respectively. Dashed lines are the classical mechanical limit. The cross marks are from Table I of Ref. 8. In (b), the SQTDH calculations with the exact extended potential (dotted, SQ) are included, but are almost indistinguishable from those with the fourth-order approximation.

tum solution is better for the asymmetric case. We note that Fig. 1(b) presents a stringent case where the wave function is delocalized over the low barrier double well. The accuracy of the fourth-order approximation will be further confirmed in Figs. 3-5.

Figure 2 shows the corresponding results for the LS potential. Note that the exact semiquantal potential is unavailable in this case, so that only the SQ4 and QM calculations are compared. The quality of the semiquantal wave packets in reproducing the quantum wave function is almost comparable to that in the case of the $\mathrm{CM}$ potential in Fig. 1. It is seen that the LS potentials in Fig. 2 are slightly narrower than the CM ones in Fig. 1. This comes from the smaller $R_{A B}$ resulted from the same parameters. For example, we used $g=1.45$ and $r_{A B}^{0}=3.0 \AA$ to generate Figs. 1(a) and 2(a), which yield $R_{A B}=2.59$ and $2.56 \AA$ for the $\mathrm{CM}$ and LS potentials, respectively. For Figs. 1(b) and 2(b), we used $g=1$ and $r_{A B}^{0}$ $=3.1 \AA$ and found $R_{A B}=2.63$ and $2.59 \AA$.

The ground state energy levels are also shown as the horizontal lines in Figs. 1 and 2. The differences between the semiquantal and quantum energies are too small to be resolved in the displayed range of the figures: The difference between the semiquantal and quantum energies, $E(\mathrm{SQ})$ 
(a)

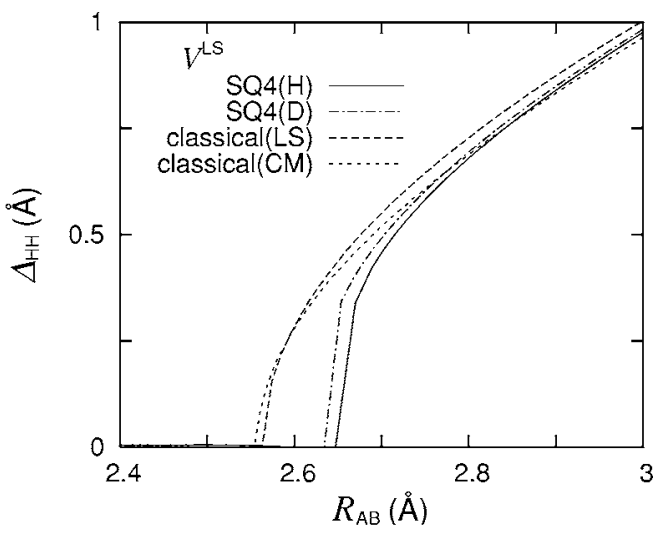

(b)

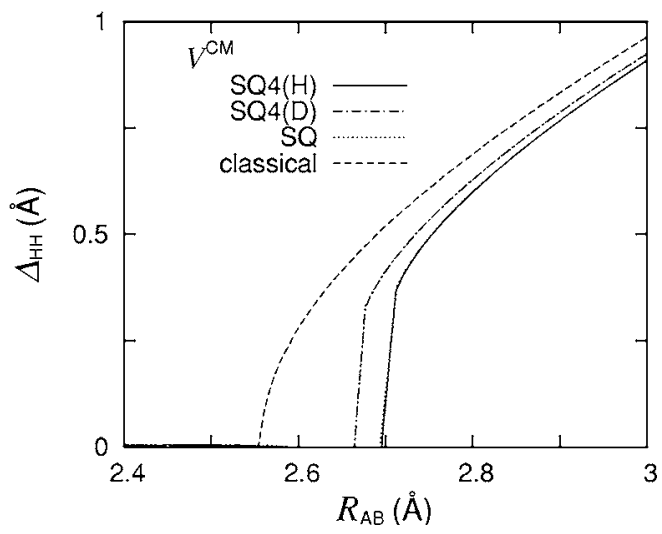

FIG. 4. Similar to Fig. 3, but the ordinate is the distance between the two hydrogen positions $\Delta_{\mathrm{HH}}$. The potential is symmetric with $g=1$.

$-E(\mathrm{QM})$, was 0.081 and $0.095 \mathrm{kcal} / \mathrm{mol}$ in Figs. 1(a) and 1 (b). With the fourth-order approximation, $E(\mathrm{SQ} 4)-E(\mathrm{QM})$ was $0.075,0.043,0.074$, and $0.094 \mathrm{kcal} / \mathrm{mol}$ in Figs. 1(a), 1(b), 2(a), and 2(b), respectively. As the consequence of the variational nature of the semiquantal approximation, the exact quantum energy is always the lowest.

\section{B. Structural correlation}

The correlation between $r_{A H}$ and $R_{A B}$ is displayed in Fig. 3. The cross marks are from Table 1 of Ref. 8 which agree well with our results in Fig. 3(a) for the LS potential with $g=1.45$. On the other hand, the corresponding results for the CM potential in Fig. 3(b) shows slower decrease along $R_{A B}$. Nonetheless, the overall qualitative behavior is similar between the two potentials. In particular, it is noted that the correlation depends strongly on the asymmetry parameter $g$. This seems to have notable implication for explaining the scattered data points observed from a variety of hydrogen bonding crystals. ${ }^{20}$ The present results suggest the necessity to reexamine the potential asymmetry for each specific system before attempting to extract a unified correlation curve from the scattered data.

In the symmetric case with $g=1, r_{A H}$ increases rapidly along with the decrease of $R_{A B}$ and then merge to the line $r_{A \mathrm{H}}=R_{A B} / 2$. This is because that the proton wave packet center approaches to the middle of $A \cdots B$ and then stays there along with the compression of the complex. Corresponding phenomenon is observed in the high-resolution neutron dif-
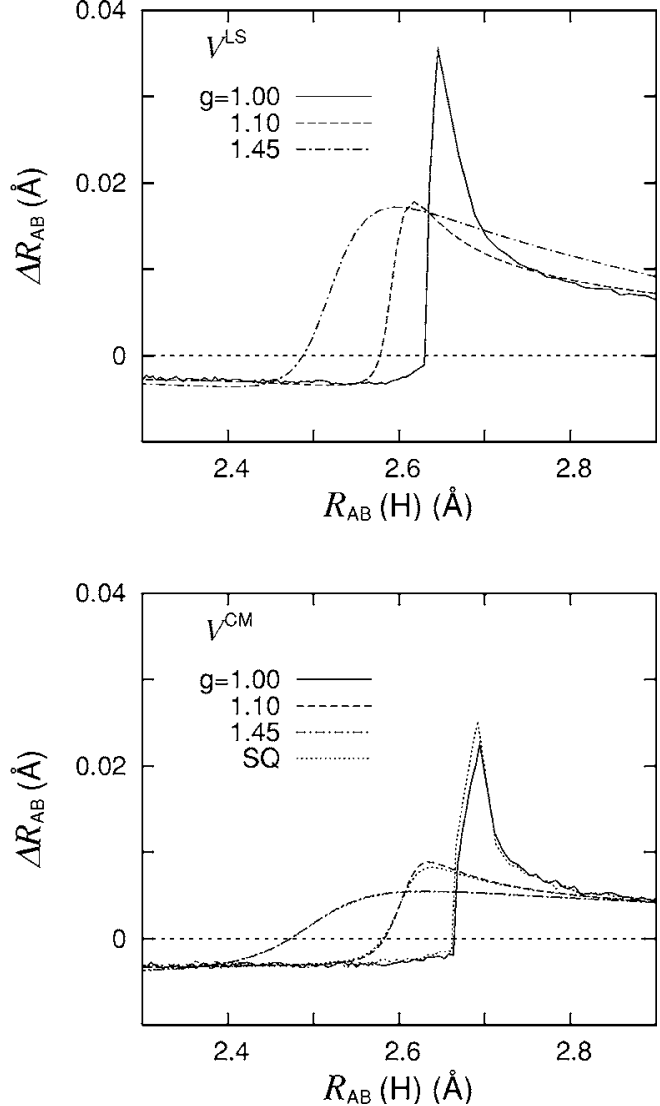

FIG. 5. The isotope shift of the heavy atoms distance $\Delta R_{A B}$ for the modified Lippincott-Schroeder (a) and the coupled Morse (b) potentials. In (b), the SQTDH calculations with the exact extended potential (dotted, SQ) are included, but the results are almost indistinguishable from those with the fourth-order approximation.

fraction measurements as a doublet proton density peaks collapsing to a single peak as $R_{A B}$ becomes shorter. We plot in Fig. 4 the distance between the two proton positions $\Delta_{\mathrm{HH}}$ along $R_{A B}$. The quantum effect is clearly seen in the existence of the critical distance $R_{A B}$ at which $\Delta_{\mathrm{HH}}$ vanishes abruptly. This contrasts with the gradual decrease of $\Delta_{\mathrm{HH}}$ in the classical limit. In the classical case, the proton positions are at the minima of the double-well potential that shift continuously along with the deformation of the potential. In contrast, the quantum wave packet does not follow the potential minima but starts to delocalize over the potential at the critical $R_{A B}$. This quantum nature is suppressed to some extent in the deuterated complex, which resulted in the smaller critical $R_{A B}$. Comparing between the LS and CM potentials, the quantum critical distances are shorter in the former whereas the classical limits are very close.

\section{Geometric isotope effect}

We define the geometric isotope effect by the difference of $R_{A B}$ between the deuterated and protonated complexes, $\Delta R_{A B} \equiv R_{A B}(\mathrm{D})-R_{A B}(\mathrm{H})$. The results are displayed in Fig. 5. Interestingly, the magnitude of the geometric isotope effect differs markedly between the LS and CM potentials such that the former exhibits about twice as large $\Delta R_{A B}$. The effect depends strongly on the asymmetry parameter $g$, with the largest effect observed for the symmetric $g=1$ case. The 
(a)
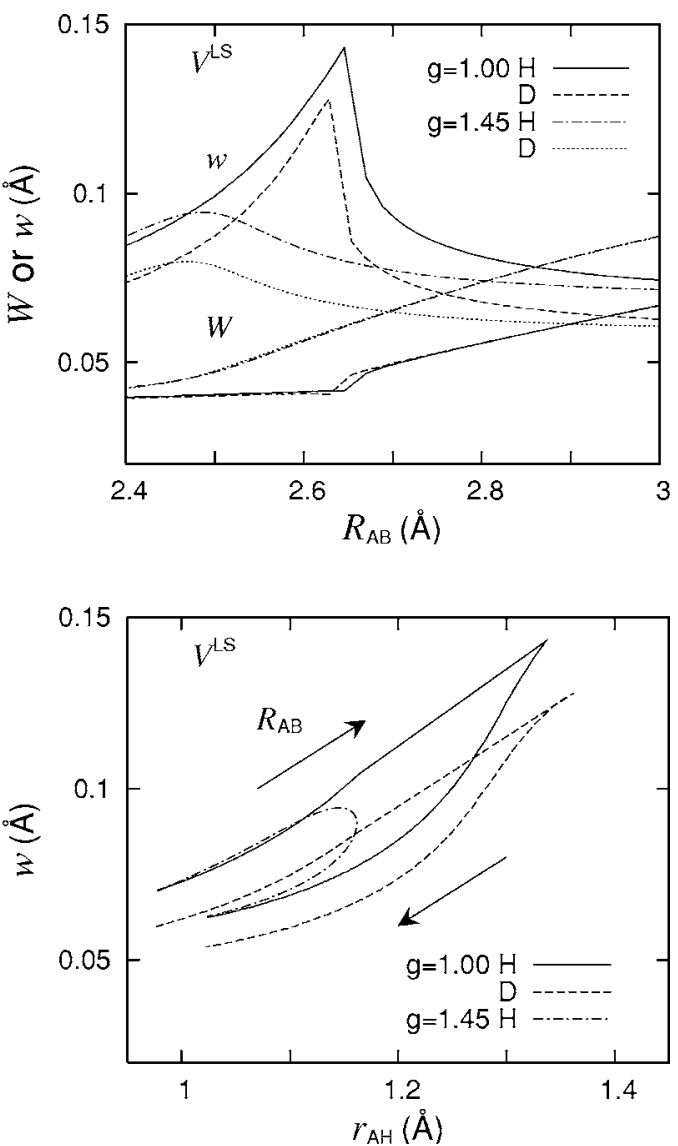

FIG. 6. The wave packet widths $w$ (upper four lines) and $W$ (lower) along $R_{A B}$ (a), and $w$ along $r_{A \mathrm{H}}$ (b), for the modified Lippincott-Schroeder potential.

small amount of the asymmetry with $g=1.1$ nearly halves the effect. The present results are in good accordance with the experimental data collected in Ref. 35 which shows that $\Delta R_{\mathrm{OO}}$ is observed when $R_{\mathrm{OO}}$ is in the range of $2.4-2.7 \AA$ and its magnitude is typically less than $0.03 \AA$. Moreover, compared to our previous investigation ${ }^{20}$ based on the $\mathrm{CM}$ potential, we seem to have approached closer to understanding the exceptional compounds such as $\mathrm{HCrO}_{2}$ and $\mathrm{HCoO}_{2}$ that exhibit much larger $\Delta R_{\mathrm{OO}}$ of around $0.06-0.10 \AA$. The present results suggests that the magnitude of $\Delta R_{A B}$ is sensitive to the small change of the potential shape. More elaborate and specific examinations, together with the possibility of collective and cooperative effects, would be needed to fully understand the effect in those exceptional compounds.

\section{Wave packet widths}

Figure 6 shows the wave packet widths $w$ and $W$ for the LS potential. Interestingly, the behavior of $w$ along $R_{A B}$ appears to be similar to that of $r_{A \mathrm{H}}$ displayed in Fig. 3. In Fig. 6(b), the correlation between $r_{A \mathrm{H}}$ and $w$ is seen to exhibit two-valued $w$ along $r_{A H}$. This comes from the two-valued $R_{A B}$ along $r_{A \mathrm{H}}$ in Fig. 3. That is, the shorter $R_{A B}$ gives the narrower potential well for the proton and squeezes the wave packet, which gives the lower branch of the $r_{A \mathrm{H}^{-}} w$ curves in Fig 6(b). For the deuterated complex, the width $w$ is smaller, but the curves from the different isotopes are nearly parallel to each other. On the other hand, the isotope effect is very

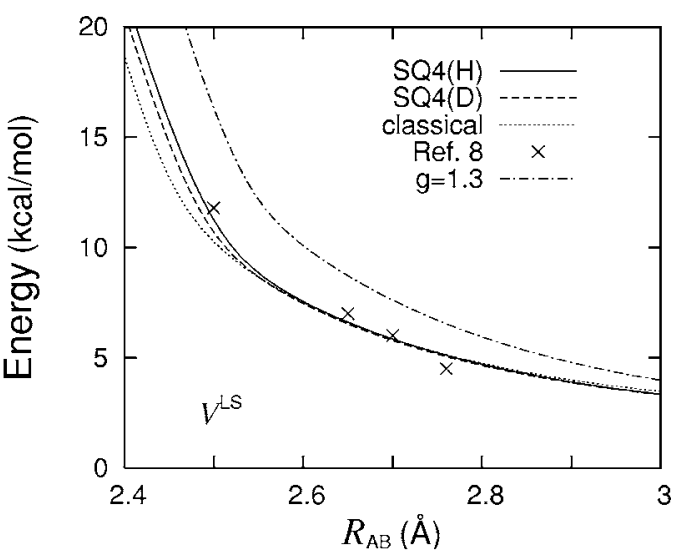

(a)

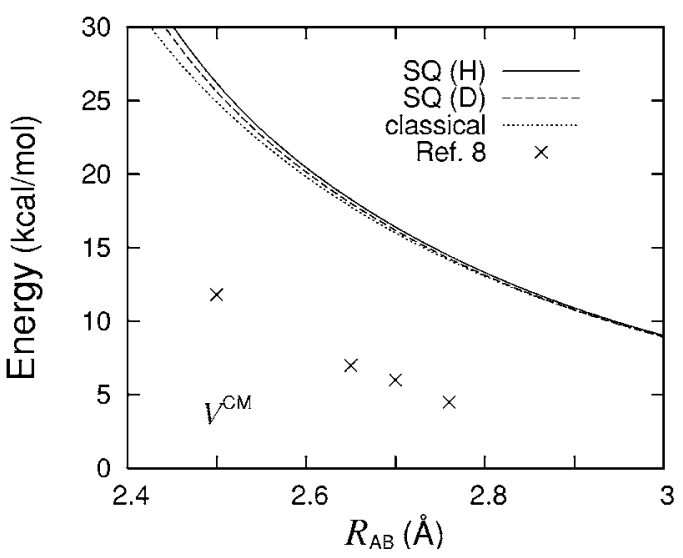

FIG. 7. The hydrogen bond formation energy from the modified LippincottSchroeder (a) and the coupled Morse (b) potentials. The former is from the fourth-order SQTDH (SQ4) and the latter is from the exact SQTDH (SQ). Solid and dashed lines are for hydrogen and deuterium, respectively. The dotted lines are the classical mechanical limit. The cross marks are the experimental data taken from Ref. 8. The asymmetry parameter $g$ is 1.45 , but the calculation with $g=1.3$ (with SQ4 and hydrogen) is also included in (a) for comparison.

small for $W$, naturally because the effect of the isotope substitution is indirect for the coordinate $R$. The width $W$ increases along $R_{A B}$, which implies that the potential along $R$ becomes shallower and the hydrogen bond weaker. The hydrogen bond strength is discussed next.

\section{E. Hydrogen bond formation energy}

Figure 7 shows the hydrogen bond formation energy as a function of $R_{A B}$. The semiquantal calculations are from the zero-point energies of the complex $A-\mathrm{H} \cdots B$ and the isolated $A-\mathrm{H}$ moiety, while the classical limit was calculated from the potential minima. As in Ref. 8, we included the thermal correction of $3 R T / 2$ for the change of translational and rotational degrees of freedom. As seen in Fig. 7(a), and noted in Sec. III C, our modified LS potential agrees well with the experimental data. The quantum effect is almost negligible in the region of $R_{A B}$ larger than around $2.5 \AA$ but becomes notable for the smaller $R_{A B}$. We primarily used the asymmetry parameter $g=1.45$, but included in Fig. 7(a) the result from $g=1.3$ for comparison. It is seen that the hydrogen bond energy depends notably on the potential asymmetry, which sounds reasonable since the asymmetry represents the difference of the proton affinity between the $A$ and $B$ atoms. 
(a)

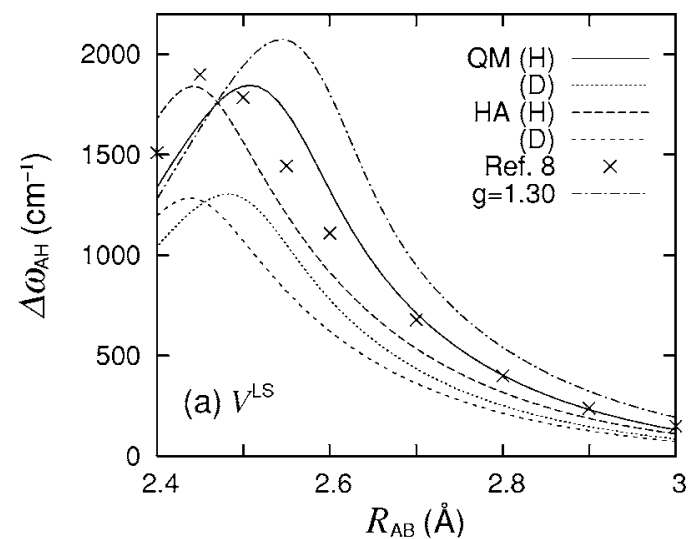

(b)

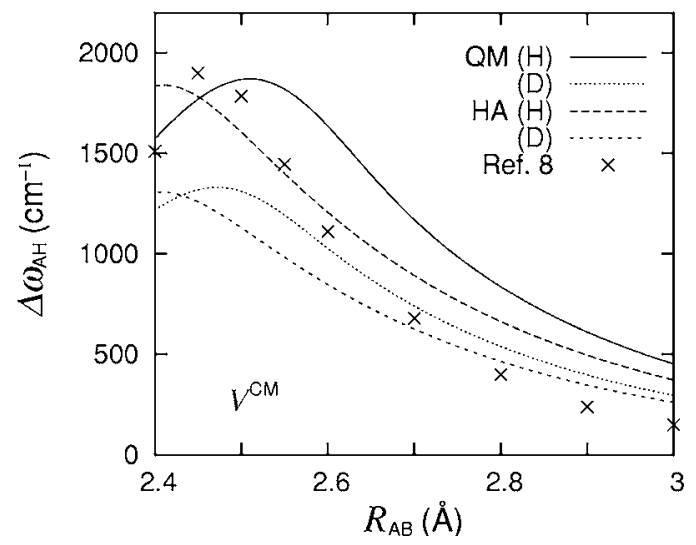

FIG. 8. The vibrational frequency shift $\Delta \omega_{A H}$ from the modified LippincottSchroeder (a) and the coupled Morse (b) potentials. QM and HA denote the quantum mechanical calculation and the harmonic approximation, respectively. The cross marks are from Table I of Ref. 8 . The asymmetry parameter $g$ is 1.45 , but the calculation with $g=1.3$ (with QM and hydrogen) is also included in (a) for comparison.

It is seen in Fig. 7(b) that the energy is largely overestimated in the CM potential. Although the better result by the LS potential is just because of the parameter optimization carried out on this potential, the difference between the two potentials is notable. Since the $V_{A B}$ part is common between the two, the difference would be the consequence of the different behavior of $V_{B \mathrm{H}}^{M}$ and $V_{B \mathrm{H}}^{L}$.

\section{F. The $A-H$ vibrational frequency shift}

We have also examined the frequency shift of $A-\mathrm{H}$ stretching vibration as a function of $R_{A B}$. Here we employed the one-dimensional Numerov method in order to obtain the first excitation energy of the $r$ vibration. ${ }^{35}$ For comparison, we also evaluated the frequency from the curvature at the bottom of the classical potential with the harmonic approximation, as has been done in Ref. 8. The results are displayed in Fig. 8. First we note the deviation between the quantum calculation and the harmonic approximation. Interestingly, they seem to overlap by introducing an appropriate scaling along the $R_{A B}$ direction. The shift is smaller by a factor of around 0.7 for the deuterated complex. Again, the shift depends on the asymmetry parameter $g$ as shown in Fig. 8(a), i.e., the small change of $g=1.45$ to 1.3 notably affects the result. We also note the non-negligible difference between the LS and CM potentials.

\section{CONCLUDING REMARKS}

A study on the structure and energetics of hydrogen bond has been presented. The computational efficiency of the SQTDH theory, providing the optimal wave packet from a straightforward minimization on the extended potential, has allowed the examination of the global nature of the hydrogen bond potentials in the broad range of structural parameters. The fourth-order expansion approximation to the extended potential was found to be accurate enough for the models studied here, which encourages us to proceed toward the study of hydrogen bond dynamics in clusters and condensed phase. Combining with the electronic structure calculations is another direction to progress, in which, for example, the non-Born-Oppenheimer electron-proton coupling and transfers $^{38-41}$ seem to represent interesting and important open questions.

\section{ACKNOWLEDGMENT}

The initial stage of this work was partially supported by The Royal Society (UK-Japan Joint Project No. 15208).

${ }^{1}$ G. C. Pimentel and A. L. McClellan, The Hydrogen Bond (Freeman, San Francisco, 1960).

${ }^{2}$ L. Pauling, The Nature of the Chemical Bond (Cornell University Press, New York, 1960).

${ }^{3}$ G. A. Jeffrey and W. Saenger, Hydrogen Bonding in Biological Structures (Springer, Berlin, 1991).

${ }^{4}$ G. A. Jeffrey, An Introduction to Hydrogen Bonding (Oxford, New York, 1997).

${ }^{5}$ K. Morokuma, Acc. Chem. Res. 10, 294 (1977).

${ }^{6}$ S. Scheiner, Hydrogen Bonding: A Theoretical Perspective (Oxford, New York, 1997).

${ }^{7}$ F. Weinhold and C. Landis, Valency and Bonding (University Press, Cambridge, 2005).

${ }^{8}$ E. R. Lippincott and R. Schroeder, J. Chem. Phys. 23, 1099 (1955).

${ }^{9}$ J. J. i Timoneda and J. T. Hynes, J. Phys. Chem. 95, 10431 (1991).

${ }^{10}$ H. Azzouz and D. Borgis, J. Chem. Phys. 98, 7361 (1993).

${ }^{11}$ A. Staib, D. Borgis, and J. T. Hynes, J. Chem. Phys. 102, 2487 (1995).

${ }^{12}$ H. J. Bakker and H. K. Nienhuys, Science 297, 587 (2002).

${ }^{13}$ A. Warshel, Computer Modeling of Chemical Reactions in Enzymes and Solution (Wiley, New York, 1991).

${ }^{14}$ K. Ando and J. T. Hynes, Adv. Chem. Phys. 110, 381 (1999), and references therein.

${ }^{15}$ M. Tachikawa, K. Mori, H. Nakai, and K. Iguchi, Chem. Phys. Lett. 290, 437 (1998).

${ }^{16}$ H. Nakai, K. Sodeyama, and M. Hoshino, Chem. Phys. Lett. 345, 118 (2001).

${ }^{17}$ A. Reyes, M. V. Pak, and S. Hammes-Schiffer, J. Chem. Phys. 123, 064104 (2005).

${ }^{18}$ K. Ando, Chem. Phys. Lett. 376, 532 (2003).

${ }^{19}$ K. Ando, J. Chem. Phys. 121, 7136 (2004).

${ }^{20}$ K. Ando, Phys. Rev. B 72, 172104 (2005).

${ }^{21}$ J. Frenkel, Wave Mechanics: Advanced General Theory (Clarendon, Oxford, 1934).

${ }^{22}$ P. Kramer and M. Saraceno, Geometry of the Time-Dependent Variational Principle in Quantum Mechanics (Springer, Berlin, 1981).

${ }^{23}$ J. Broeckhove, L. Lathouwers, E. Kesteloot, and P. van Leuven, Chem. Phys. Lett. 149, 547 (1988).

${ }^{24}$ F. Arickx, J. Broeckhove, E. Kesteloot, L. Lathouwers, and P. van Leuven, Chem. Phys. Lett. 128, 310 (1986).

${ }^{25}$ Y. Tsue, Prog. Theor. Phys. 88, 911 (1992).

${ }^{26}$ A. K. Pattanayak and W. C. Schieve, Phys. Rev. E 50, 3601 (1994).

${ }^{27}$ O. V. Prezhdo, J. Chem. Phys. 117, 2995 (2002).

${ }^{28}$ E. R. Lippincott and R. Schroeder, J. Chem. Phys. 23, 1131 (1955).

${ }^{29}$ M. S. Child, Molecular Collision Theory (Dover, New York, 1996).

${ }^{30}$ E. R. Lippincott, J. Chem. Phys. 21, 2070 (1953).

${ }^{31}$ E. R. Lippincott, J. Chem. Phys. 26, 1678 (1956). 
${ }^{32}$ W. L. Press, B. P. Flannery, S. A. Teukolsky, and W. T. Vetterling, Numerical Recipes: The Art of Scientific Computing (Cambridge University Press, New York, 1989).

${ }^{33}$ B. R. Johnson, J. Chem. Phys. 67, 4086 (1977).

${ }^{34}$ J. Römelt, Chem. Phys. Lett. 74, 263 (1980).

${ }^{35}$ Alternatively, we could have applied the quantization procedure that makes use of the geometrical phase calculated from the action in the extended phase space (Refs. 36 and 37).
${ }^{36}$ A. K. Pattanayak and W. C. Schieve, Phys. Rev. E 56, 278 (1997).

${ }^{37}$ R. G. Littlejohn, Phys. Rev. Lett. 56, 2000 (1986).

${ }^{38}$ R. I. Cukier and D. Nocera, Annu. Rev. Phys. Chem. 49, 337 (1998).

${ }^{39}$ H. Nakai and K. Sodeyama, J. Chem. Phys. 118, 1119 (2003).

${ }^{40}$ T. Ishimoto, M. Tachikawa, H. Tokiwa, and U. Nagashima, Chem. Phys. Lett. 314, 231 (2005).

${ }^{41}$ C. Swalina, M. V. Pak, and S. Hammes-Schiffer, J. Chem. Phys. 123, 014303 (2005). 